\title{
Supersymmetric frontiers and beyond
}

Towards the ultimate theory of physical laws.

\section{Supersymmetry: Unveiling the Ultimate Laws of Nature \\ by Gordon Kane \\ Perseus: 2000. 199 pp. \$26, £15.95}

\section{Gian Francesco Giudice}

The modern approach taken by physicists in attempting to understand the ultimate laws of nature has been shaped by Einstein's general relativity theory. In this, any concept of force at a distance is abandoned and symmetry emerges as a fundamental principle. Einstein had the intuition to see that a full understanding of the physical laws requires a unified treatment for gravity and electromagnetism, the two forces known at the time.

This idea of unification is still central in contemporary theoretical physics. But two new forces have since been discovered in the microscopic world: the strong force, responsible for the cohesion of nuclei, and the weak force, responsible, for instance, for neutron decay. These forces are now understood in terms of simple symmetry principles and are well described by what is called the Standard Model. Actually, the Standard Model partially achieves Einstein's dream of unification, because it identifies electromagnetic and weak forces as a single entity. As a result, the Standard Model gives a superbly successful description of particle interactions that has been verified experimentally with meticulous precision.

The Standard Model is the magnificent tool that allows today's physicists to address the most fundamental questions about the microscopic structure of matter and the evolution of the Universe, starting from a billionth of a second after the Big Bang. Nonetheless, it cannot be the ultimate theory of physical laws. As Gordon Kane states in his book, "someone who probes and studies a watch not only can describe the workings of that watch but also can say why the watch works". Physicists not only want to describe how the Universe works, but also want to understand why it works in that way. In other words, they want to derive the ultimate theory underlying the Standard Model, achieve a unification of all forces, and identify the fundamental symmetry principles that determine the laws of nature. As explained by Kane, the achievement of this goal may not be so far away, and supersymmetry may be a key that will lead us to it.

Supersymmetry is an unusual symmetry under which particles possessing integer spin (bosons) and half-integer spin (fermions) are exchanged. If supersymmetry holds, the laws of particle physics will be unaffected by this special interchange of bosons and fermions. Because, among known particles, bosons are carriers of force and fermions describe matter, supersymmetry may tell us something about the relation between force and matter. In a supersymmetric world, the concept of space-time is overtaken by a new physical entity: superspace. Superspace is an extension of ordinary space-time in which new dimensions open up. These dimensions have very peculiar mathematical properties, because their coordinates are not usual numbers, but anticommuting variables (in other words, the product of two such numbers $A$ and $B$ satisfies the unusual property $A \times B=-B \times A$ ).

All this may sound very abstract. Indeed, the mathematical details of the theory are quite involved. Moreover, this is not a settled scientific theory, but a field of research in which progress is ongoing. This is, I believe, the most fascinating aspect of Kane's book. A reader with no prior knowledge of particle physics is led, always with lucid and clear explanations, from a simple introduction to the particle physics world to the frontiers of today's research. The puzzles and problems that physicists are trying to tackle are clearly revealed. Not only are readers confronted with the most recent theoretical developments in particle physics, but they can also see how physicists think in their everyday work, how they try to identify the appropriate scientific questions and proceed to find answers.
One cannot expect to learn about a scientifically established theory here. On the other hand, one can learn how basic research works and how a scientific hypothesis is developed and formulated. Of course, a scientifically sound hypothesis needs experimental scrutiny, and the theory of supersymmetry is ready for it. Kane explains how future collider experiments will be able to test the predictions made by supersymmetry. This is also a useful excuse for taking the reader on a fascinating tour of the largest accelerators in the world, in which elementary particles collide at extremely high energies and the debris products are identified and measured by sophisticated giant detectors. Most notably, the ultimate answer on the fate of supersymmetry should come from the LHC, the Large Hadron Collider under construction at CERN, the European Laboratory for Particle Physics, which will generate the highest energy collisions ever achieved.

This book gives a fascinating account of the theoretical ideas behind supersymmetry and the experimental programme aimed at its confirmation. It is told by someone who has contributed deeply to the development of the field. Kane is such an enthusiastic guide that the reader has the impression of almost participating in the research. As the tension builds, one wants to know how the story ends. But the final chapter has not yet been written. Only future research in theoretical



\section{Filling space-time}

Carpets can have many forms of symmetry, although perhaps not of the super variety. One example is shown above, from western Turkestan.
Photographs of oriental carpets of many designs and patterns are to be found in The Carpet by Enza Milanesi (Tauris Parke/Firefly, $\mathfrak{E 2 9 . 5 0 / \$ 5 0 ) . ~}$ 
and experimental particle physics will reveal whether supersymmetry can bring us nearer to the ultimate laws of nature.

Gian Francesco Giudice is in the Theoretical Physics Division, CERN, CH-1211 Geneva 23, Switzerland.

\section{The counting-house called to account}

\section{How to Build a Mind}

by Igor Aleksander

Weidenfeld \& Nicolson: 2000.181 pp. $£ 14.99$

\section{Steve Blinkhorn}

Is the brain the organ of the mind? Aristotle thought it was there to cool the blood, a function it performs admirably in cold climates - witness the invention of the hat. But the common view, informed by 400 years of philosophy merging into 150 years of neurology, is that, as a settled matter of fact, the brain is the organ of the mind, with the only real puzzle being how the brain generates consciousness.

What a peculiar and uninformed position to arrive at. It suggests that people are, in a psychological sense, no more than brains on legs. The fact that adrenals, gonads and thyroid profoundly influence our conscious experience, and that sensation without sense organs is wildly discrepant and disruptive (as in the case of phantom limbs), are set aside in the hunt for the rational singularity of mind at the centre of experience.

Descartes started it, but then Locke got carried away with enthusiasm for the camera obscura, one of the original technological metaphors that supply psychologists with models when they have run out of ideas. Since then, we have been stuck with the idea that vision is the primary feedstock of consciousness, and that vision involves an internal observer making sense of what were once called 'sense data'. Anyone who has at some time lost proprioceptive and tactile sensory input over a significant part of their body knows what a loss it is, and what an important part of the sense of self it contributes. We are, as whole organisms, extended in space.

The science fiction notion of a disembodied brain floating in a nutrient solution, connected to artificial sensors by coiled cables (for some reason they are always coiled, even though the brain is immobile), is not futuristic, it is medieval. A serious examination of the functions that a disembodied immortal soul might be able to support suggests a limited range centred on incessant theological contemplation.

A disembodied brain would be little better off. Consider that, in the economy of a whole individual, the brain maybe no more than the counting-house. Accountants, the inhabitants of counting-houses, often suffer from the illusion that it is their efforts, and not those of the production-workers, sales staff and designers, that sustain the life of the enterprise. To be sure, few businesses would survive long without an effective finance function, but on its own it is utterly worthless.

Igor Aleksander has made a career out of swimming against fashionable intellectual currents so far as the notion of consciousness is concerned. When artificial intelligence was in fashion (knowledge-based systems, inference engines, 'expert systems', and the like), he was building machines on a shoestring that had emergent properties, and publishing in unfashionable places.

He works on the principle that, to make a conscious machine, you have to make a machine that is complex enough to generate its own kind of consciousness. Its desires and needs will be determined by its physical nature, not a programmer's idea of what consciousness ought to be like. The critical point comes when a machine generates internal representations of the world that are, to use Aleksander's phrase, "egocentred": the machine represents itself to itself as a whole vis-à-vis a stable environment. You don't write a program to generate this style of representation: you build multiple, loosely coupled, asynchronously interacting subsystems that converge on it.

But it is appallingly difficult to capture these sorts of ideas in an accessible book and, although How to Build a Mind is stuffed with fine ideas, it is not a fine book. Part of the problem is the mix of methods and, in particular, the use of invented dialogues. Plato is to blame for the seductive appeal these have for authors (although, of course, he blamed Socrates); they got Galileo into awful trouble with the pope; and Aleksander isn't quite up to the literary style of Plato or the organizing genius of Galileo. Mostly, Aleksander's dialogues involve historical figures in philosophy, but they are historically unconvincing, and caricature, rather than expound, the distinctive viewpoints of their subjects. I don't know how to do it better, but it isn't done well here.

Aleksander has a point of view that stems from his discipline: engineers are people who make things that work. If he can make a machine that generates self-representations able to distinguish consistently between self and non-self, why should this not constitute a kind of consciousness? Picking up on George Kelly's dictum that "a person's psychological processes are channelised by the ways in which he anticipates events", a cat showing signs of embarrassment is surely to that extent conscious, and might not even a bee, however dimly, be representing to itself, somewhere in its 900,000 neurons, the flight path back from a food source?

Debates on mind, brain and consciousness can suffer from a lack of attention to proper definition. Your notion of consciousness may differ from mine mostly in terms of our contemplation of the opposite. There are many ways of being unconscious, from the comatose to the Freudian, and we lack good distinctive terms to delineate awareness of the world, awareness of self as persistent over time and space, and the ability to exercise critical self-examination.

The overwhelming tendency in the history of philosophy, and now in the blinkered search for the means by which consciousness is generated by the brain, has been to focus on the most abstruse levels of mental functioning, such as doing mathematics, playing chess, understanding complex abstract language. But none of us got where we are without a long period of evolving consciousness and special training in a period called 'childhood', and most people spend absolutely none of their time doing any of these things anyway. Aleksander's machines have barely had the opportunity to get over their birth pangs by comparison.

What lies in the future for them is hinted at in one of the more startling of the insights that pepper How to Build a Mind: "if there is no perception in any sensory channel, the inner networks can fall into 'attractors' ... If this occurs during sleep, it is called dreaming."

What price a computer that dreams, or better still, that is a lucid dreamer?

Steve Blinkhorn is at Psychometric Research and Development Ltd, Brewmaster House,

The Maltings, St Albans AL1 3HT, UK.

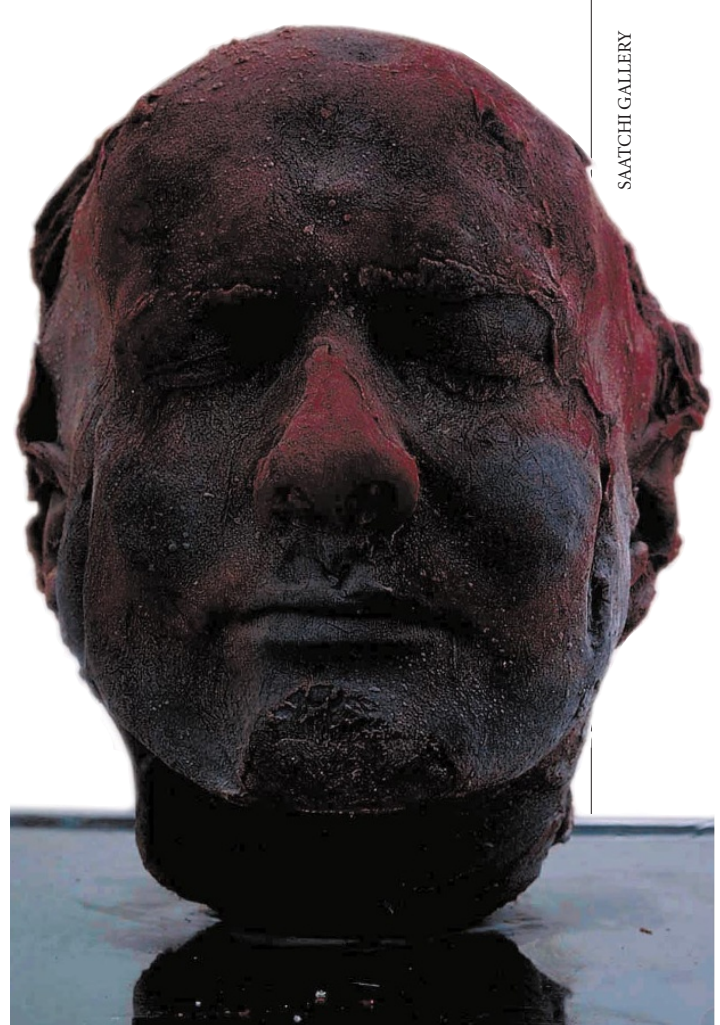

Sanguine contemplation: sculptor Marc Quinn's Self, cast in his own blood.

NATURE $\mid$ VOL 407 5 OCTOBER 2000 | www.nature.com 